Mon. Not. R. Astron. Soc. 000, 000-000 (0000) Printed 25 December $2019 \quad$ (MN LATEX style file v2.2)

\title{
HD 93795: a late-B supergiant star with a square circumstellar nebula
}

\author{
V. V. Gvaramadze, ${ }^{1,2 \star}$ A. Y. Kniazev, ${ }^{3,4,1}$ N. Castro ${ }^{5}$ and I. Y. Katkov ${ }^{1,6}$ \\ ${ }^{1}$ Sternberg Astronomical Institute, Lomonosov Moscow State University, Universitetskij Pr. 13, Moscow 119234, Russia \\ ${ }^{2}$ Space Research Institute, Russian Academy of Sciences, Profsoyuznaya 84/32, 117997 Moscow, Russia \\ ${ }^{3}$ South African Astronomical Observatory, PO Box 9, 7935 Observatory, Cape Town, South Africa \\ ${ }^{4}$ Southern African Large Telescope Foundation, PO Box 9, 7935 Observatory, Cape Town, South Africa \\ ${ }^{5}$ Leibniz-Institut für Astrophysik Potsdam (AIP), An der Sternwarte 16, 14482, Potsdam, Germany \\ ${ }^{6}$ New York University Abu Dhabi, Saadiyat Island, PO Box 129188, Abu Dhabi, UAE
}

Accepted 2019 December 21. Received 2019 December 18; in original form 2019 December 04

\begin{abstract}
We report the discovery of a square axisymmetric circumstellar nebula around the emission-line star HD 93795 in archival Spitzer Space Telescope $24 \mu \mathrm{m}$ data. We classify HD 93795 as an B9 Ia star using optical spectra obtained with the Southern African Large Telescope (SALT). A spectral analysis carried out with the stellar atmosphere code FASTWIND indicates that HD 93795 only recently left the main sequence and is evolving redward for the first time. We discuss possible scenarios for the origin of the nebula and suggest that HD 93795 was originally a binary system and that the nebula was formed because of merger of the binary components. We also discuss a discrepancy between distance estimates for HD 93795 based on the Gaia data and the possible membership of this star of the Car OB1 association, and conclude that HD 93795 could be at the same distance as Car OB1.
\end{abstract}

Key words: stars: abundances - circumstellar matter - stars: emission-line, Be stars: individual: HD 93795 - stars: massive - supergiants

\section{INTRODUCTION}

Inspection of archival data from infrared (IR) sky surveys carried out with the Spitzer Space Telescope (Werner et al. 2004) and the Wide-field Infrared Survey Explorer (WISE; Wright et al. 2010) led to the discovery of numerous compact $(\lesssim 1$ arcmin in diameter) nebulae of various shapes (e.g. Gvaramadze, Kniazev \& Fabrika 2010; Gvaramadze et al. 2012). Spectroscopic follow-up of central stars of these nebulae revealed that many of them belong to rare types of massive stars, such as the luminous blue variables and WolfRayet stars, displaying rich emission spectra (e.g. Kniazev \& Gvaramadze 2015; Gvaramadze \& Kniazev 2017 and references therein). A significant part of the nebulae, however, was found to be associated with blue supergiant stars whose spectra are either free of emission lines or have mostly the $\mathrm{H} \alpha$ line in emission. A spectral analysis of some of these stars showed that they only recently left the main sequence, meaning that massive stars can produce circumstellar nebulae not only at advanced, but also during early stages of their evolution (e.g. Lamers et al. 2001; Smartt et al. 2002;

\footnotetext{
* E-mail: vgvaram@mx.iki.rssi.ru
}

Hendry et al. 2008; Gvaramadze et al. 2014, 2015, 2019a; Mahy et al. 2016).

Although the mechanisms responsible for the origin and shaping of circumstellar nebulae are not fully understood, the axial or bipolar geometry of many of them strongly suggests that binarity or rapid rotation of their underlying stars can play a decisive role in their formation. Detection of new examples of axially-symmetric circumstellar nebulae associated with relatively unevolved massive stars could provide important clues about their origin. In this paper, we report the discovery of such a nebula around the late-B supergiant star HD 93795.

In Section2, we review the existing data on HD 93795 and present the nebula. Section 3 describes our long-slit and fibre-fed échelle spectroscopic observations of HD 93795. The spectral classification of HD 93795 and the results of modelling of its spectrum with the stellar atmosphere code FASTWIND are given in Section 4. In Section 5, we report the detection of shocked gas within and beyond the confines of the nebula. In Section 6] we discuss possible mechanisms for the origin of the nebula. We summarise in Section 7 

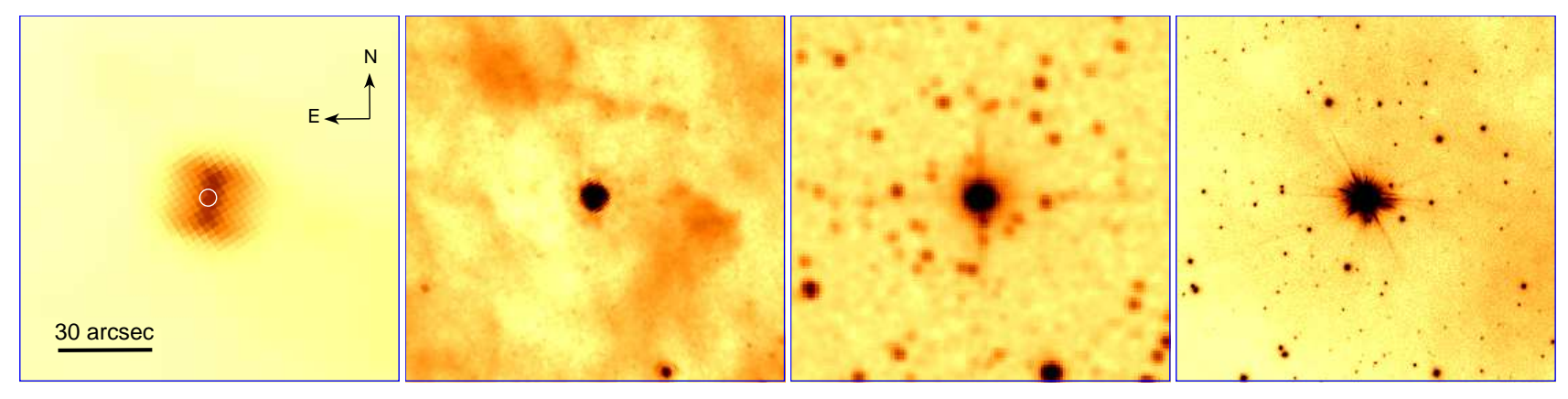

Figure 1. From left to right: Spitzer MIPS $24 \mu \mathrm{m}$ and IRAC $8 \mu \mathrm{m}, 2 \mathrm{MASS} K_{\mathrm{S}}$ and VPHAS+ H $\alpha$ images of HD 93795 and its circumstellar nebula. A white circle in the MIPSE image shows the position of HD 93795. The orientation and the scale of the images are the same. At the distance of $5 \mathrm{kpc}, 30$ arcsec correspond to $\approx 0.72 \mathrm{pc}$.

\section{A SQUARE INFRARED NEBULA AROUND HD 93795}

HD 93795 is an emission-line star (MacConnell 1981) located in the direction of the giant $\mathrm{HII}$ region NGC 3372 (also known as Carina Nebula), hosting the famous luminous blue variable star $\eta$ Car and several massive star clusters (e.g. Feigelson et al. 2011). HD 93795 was previously classified as A0 Ia (Hoffleit 1956), B6 I (Stephenson \& Sanduleak 1971), B9 Iab (Humphreys 1973), and A2/3p: shell: (Houk \& Cowley 1975).

The line of sight to HD93795 is tangential to the Sagittarius-Carina spiral arm, meaning that this star is located somewhere between $\approx 2$ and $7 \mathrm{kpc}$ from the Sun. According to Humphreys (1978), HD 93795 is a member of the Car OB1 association, which is located at the distance of $2.3 \pm 0.2 \mathrm{kpc}$ (Lim et al. 2019). The Gaia second data release (DR2; Gaia Collaboration et al. 2018) parallax of HD 93795 of $0.1975 \pm 0.0356$ mas places this star at a distance of $d=5.06_{-0.77}^{+1.11} \mathrm{kpc}$. A somewhat shorter distance to HD 93795 of $d=4.32_{-0.59}^{+0.80} \mathrm{kpc}$ was derived from the DR2 data by Bailer-Jones et al. (2018) by using a Bayesian prior knowledge approach. Both these distances are much larger than the distance to Car OB1, suggesting that HD 93795 might be a background star projected by chance on Car OB1. In what follows, we adopt a distance of $d=5 \pm 1 \mathrm{kpc}$ for HD 93795 . Still, due to the zero-point problem in the Gaia DR2 parallaxes (Lindegren et al. 2018; Stassun \& Torres 2018; Zinn et al. 2019) and because the parallax measurements could be affected by possible binarity of HD 93795 (suggested by radial velocity variability of the star; see below and Section 4.2), one cannot exclude the possibility that this star is located at the same distance as Car OB1 (cf. Sections 4.3 and 5). We will consider this possibility as well.

In literature we found three measurements of the heliocentric radial velocity, $v_{\mathrm{r}, \mathrm{hel}}$, of HD 93795. Two of them, $-20.7 \pm 2.2 \mathrm{kms}^{-1}$ and $-4.1 \pm 1.5 \mathrm{kms}^{-1}$, are given in Humphreys (1973), and the third one, $-12.4 \mathrm{~km} \mathrm{~s}^{-1}$, in Humphreys (1978). We compare them with our own measurements and discuss in Section 4.2 .

In Table 1 we provide a short summary of the properties of HD 93795. The spectral type is based on our observations. The coordinates and the $B$ and $V$ magnitudes are from the Tycho Input Catalogue (Egret et al. 1992). The $J$ and $K_{\mathrm{s}}$ magnitudes are from the Two-Micron All Sky Sur-
Table 1. Properties of HD 93795.

\begin{tabular}{lc}
\hline Spectral type & B9 Ia \\
RA(J2000) & $10^{\mathrm{h}} 48^{\mathrm{m}} 23^{\mathrm{s}} 10$ \\
Dec.(J2000) & $-59^{\circ} 32^{\prime} 26^{\prime \prime} 9$ \\
$l$ & 287.9038 \\
$b$ & -0.3083 \\
$B$ (mag) & $9.30 \pm 0.01$ \\
$V$ (mag) & $8.50 \pm 0.01$ \\
$J$ (mag) & $6.43 \pm 0.02$ \\
$K_{\mathrm{s}}$ (mag) & $5.90 \pm 0.04$ \\
\hline
\end{tabular}

vey (2MASS; Skrutskie et al. 2006) All-Sky Catalog of Point Sources (Cutri et al. 2003).

The nebula around HD 93795 was discovered in the search for bow-shock-producing stars running away from massive star clusters, including those in the Carina Nebula (for motivation of this search see Gvaramadze \& Bomans 2008). We found it in the MIPS (Multiband Imaging Photometer for Spitzer; Rieke et al. 2004) $24 \mu \mathrm{m}$ image obtained in the framework of the Spitzer survey of the Carina Nebula (program ID 30848; N. Smith, PI). The nebula is not visible at shorter wavelengths, nor in the IRAC (Infrared Array Camera; Fazio et al. 2004) 8, 5.8, 4.5 and $3.6 \mu \mathrm{m}$ images from the Spitzer Vela-Carina survey (program ID 40791; S.R. Majewski, PI), nor in the $\mathrm{H} \alpha$ images from the SuperCOSMOS H-alpha Survey (Parker et al. 2005) and the VST Photometric H $\alpha$ Survey of the Southern Galactic Plane and Bulge (VPHAS+; Drew et al. 2014).

In the $24 \mu \mathrm{m}$ image the nebula appears as a square of $\approx 30$ arcsec side with a bright ridge in the middle and two symmetric flanks perpendicular to the ridge (see Fig.1). The linear size of the nebula scales with the distance as $\approx 0.72(d / 5 \mathrm{kpc}) \mathrm{pc}$. The geometry of the nebula suggests that we deal with a disc-like structure viewed almost edgeon (the ridge) and a bipolar outflow perpendicular to the disc plane. In this connection, we note that Heiles (2000) measured polarization towards HD 93795 of $2.21 \pm 0.40$ per cent with a position angle of $\mathrm{PA}=95.0^{\circ} \pm 5.2^{\circ}$ (measured from north to east). If a significant fraction of the polarization is intrinsic to the star itself, then this would imply the presence of a flattened circumstellar structure around the star with the major axis roughly parallel to the ridge in the IR nebula (whose $\mathrm{PA}$ is $\approx 168^{\circ}$ ). 


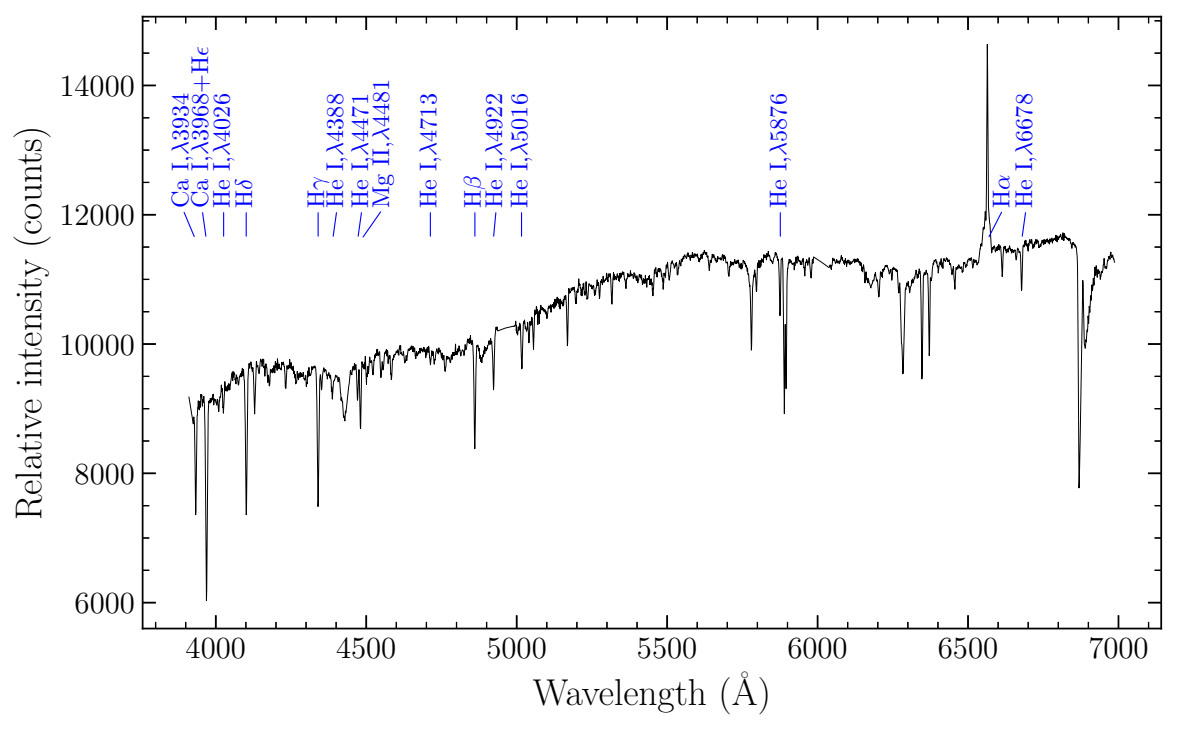

Figure 2. The observed RSS spectrum of HD 93795 obtained on 2015 July 3, with the main lines indicated. The gaps in the spectrum at $\approx 4900$ and $6000 \AA$ are due to gaps between CCDs.

\section{SPECTROSCOPIC OBSERVATIONS}

We observed HD 93795 for the first time on 2013 April 26 as part of our ongoing program of spectroscopic follow-up of central stars of IR nebulae detected with Spitzer and WISE (e.g. Gvaramadze et al. 2010, 2012). The observation was carried out with the Robert Stobie Spectrograph (RSS; Burgh et al. 2003; Kobulnicky et al. 2003) at the Southern African Large Telescope (SALT; Buckley, Swart \& Meiring 2006; O'Donoghue et al. 2006). The PG900 grating was used along with a $8 \operatorname{arcmin} \times 1.25$ arcsec slit, covering the spectral range of $4200-7300 \AA$ with a final reciprocal dispersion of $0.97 \AA$ pixel $^{-1}$. The spectral resolution FWHM was $4.47 \pm 0.25 \AA$. Three science spectra were taken with exposure times of 1,10 and 60 s to avoid possible saturation of the $\mathrm{H} \alpha$ emission line. An Xe lamp arc spectrum was taken immediately after the science frame. The slit was oriented at $\mathrm{PA}=170^{\circ}$. The seeing during the observations was 1.5 arcsec.

HD 93795 was observed for the second time with RSS on 2015 July 3. Again the PG900 grating was used with the $8 \operatorname{arcmin} \times 1.25 \operatorname{arcsec}$ slit, but this time with the spectral setup covering the spectral range of $3900-7000 \AA$ with a final reciprocal dispersion of $0.97 \AA$ pixel $^{-1}$. The spectral resolution FWHM was $4.45 \pm 0.23 \AA$. Two spectra were taken with exposures of 15 and $180 \mathrm{~s}$ (in both cases the slit was oriented at $\mathrm{PA}=80^{\circ}$ ). The seeing during these observations was about 2.0 arcsec. The short exposure was used to search for possible major changes in the spectrum of the star, while the longer exposure was intended to search for nebular emission around the star.

A spectrum of an Ar comparison arc was obtained immediately after the observations to calibrate the wavelength scale. For the relative flux calibration the spectrophotometric standard stars EGGR 21 (Baldwin \& Stone 1984) and LTT 9239 (Hamuy et al. 1992, 1994) were observed with the same spectral set-ups during nearest twilights as a part of the SALT calibration plan.

The RSS spectra were first reduced with the SALT science pipeline (Crawford et al. 2010) and then further reduced in the way described in Kniazev et al. (2008). The spectrum obtained in 2013 was presented in Kniazev \& Gvaramadze (2015), where we tentatively classified HD 93795 as an A star (no luminosity class was derived at that time). The only emission line detected in this spectrum is $\mathrm{H} \alpha$. The short-exposure spectrum of HD 93795 from 2015 is presented in Fig.2 The comparison of the RSS spectra showed that the intensity of the $\mathrm{H} \alpha$ line has increased in 2015 by about 10 per cent, while its overall shape is slightly changed (cf. Section 4.2). The analysis of the long-exposure spectrum led to the detection of shocked gas within and beyond the confines of the IR nebula (see Section 5).

For spectral modelling and to search for possible radial velocity variability, we obtained two spectra of HD 93795 with the High Resolution Spectrograph (HRS; Barnes et al. 2008; Bramall et al. 2010, 2012; Crause et al. 2014), which is also mounted on SALT. The spectra were taken in the medium resolution (MR) mode on 2018 December 21 and 2019 February 5 with the exposure time of each observation of $210 \mathrm{~s}$. The seeing during these observations was 1.8 and 1.7 arcsec, respectively.

The HRS is a dual beam, fibre-fed échelle spectrograph. In the MR mode it has 2.23 arcsec diameter for both the object and sky fibres providing a spectrum in the blue and red arms over the spectral range of $\approx 3700-8900 \AA$ with resolving power of $R \approx 40000$ (Kniazev et al. 2019). Both the blue and red arm CCDs were read out by a single amplifier with a $1 \times 1$ binning. A spectrum of a ThAr lamp and three spectral flats were obtained in the MR mode during a weekly set of HRS calibrations. For the relative flux calibration spectrophotometric standard stars from the list in Kniazev (2017) were observed once per week as a part of the SALT HRS calibration plan. During our observations the star ksi02 Cet (Turnshek et al. 1990) was used as a standard.

The spectra were first reduced with the SALT science 
pipeline (Crawford et al. 2010) and then further reduced as described in Kniazev, Gvaramadze \& Berdinikov (2016).

We also retrieved and co-added processed spectra of HD 93795 from the European Southern Observatory (ESO) data archive. These spectra were obtained on 2014 April 26 under programme ID 092.A-9020(A) with the highresolution $(R=48000)$ échelle spectrograph FEROS (Fibrefed Extended Range Optical Spectrograph; Kaufer et al. 1999) at the 2.2-m Max Planck Gesellschaft telescope (La Silla). Eight spectra covering the spectral range of $\approx 3500-$ $9200 \AA$ were taken with a total exposure time of $2400 \mathrm{~s}$. The seeing in these observations was $\approx 1.4-1.6$ arcsec. The obtained spectra were reduced with the ESO pipeline.

\section{SPECTRAL ANALYSIS}

\subsection{Spectral classification}

The spectrum of HD 93795 is dominated by the H I and He I lines (see Fig.22). The $\mathrm{H} \alpha$ line is in emission and shows a complex profile (see below). In the HRS spectra there are several weak emission lines of Fe II at 7496, 7513, 7732, 7755, 7789 and $7848 \AA$. The $\mathrm{Mg}$ II $\lambda 4481 / \mathrm{He} \mathrm{I} \lambda 4471$ and Fe II $\lambda 4233 /$ Si II $\lambda \lambda 4128,4132$ intensity ratios of $>1$ and $<1$, respectively, imply that HD 93795 is of B9 spectral type (see Evans et al. 2004), which agrees with the spectral classification by Humphreys (1973).

To derive the luminosity class, we use the Keenan \& Hynek (1950) calibration between equivalent width (EW) of the O I $\lambda \lambda 7772-5 \AA$ triplet and the luminosity class. With $\mathrm{EW}(\mathrm{OI})=1.80 \pm 0.01 \AA$ (measured in the HRS spectra), one finds the luminosity class Ia, meaning that HD 93795 is more luminous then it is suggested by the luminosity class Iab given in Humphreys $(1973,1978)$.

\subsection{Spectral modelling and stellar parameters}

The three spectra of HD 93795 were corrected for heliocentric velocity and radial velocity variations (see below), and co-added to increase the signal-to-noise ratio. The resulting spectrum was analysed using the stellar atmosphere code FASTwind (Santolaya-Rey, Puls \& Herrero 1997; Puls et al. 2005; Rivero González et al. 2012). FASTwIND calculates the atmosphere and line formation in spherical symmetry, with an explicit treatment of the stellar wind and taking into account non-local thermodynamic equilibrium (non-LTE).

The quantitative analysis was carried out in two steps. First, several key lines of HI, HeI and Si II were compared to a synthetic FASTWIND grid tailored for the analysis of Btype supergiants. The quantitative analysis follows the $\chi^{2}$ approach described in Castro et al. (2012). A full description of the stellar parameters covered in the grid and the list of lines used in the analysis can also be found in Castro et al. (2012).

Second, and based on the stellar parameters obtained in the first step (see Table2), a new FASTwind sub-grid was build for different chemical abundances of $\mathrm{Mg}, \mathrm{C}, \mathrm{Si}, \mathrm{O}$ and $\mathrm{N}$. The grid ranged \pm 2 dex around the cosmic abundance standard (CAS) in the solar neighborhood (Nieva \& Przybilla 2012) in steps of 0.1 dex. The grids was also built for different microturbulence velocities, $v_{\text {mic }}$, exploring values
Table 2. Stellar parameters and non-LTE metal abundances (by number) of HD 93795. The cosmic abundance standard in the solar neighbourhood (Nieva \& Przybilla 2012) is given in brackets for reference.

\begin{tabular}{lc}
\hline$T_{\text {eff }}(\mathrm{kK})$ & $11 \pm 1$ \\
$\log g(\mathrm{cgs})$ & $1.6 \pm 0.1$ \\
$v_{\text {mic }}\left(\mathrm{km} \mathrm{s}^{-1}\right)$ & $13 \pm 2$ \\
$v_{\operatorname{mac}}\left(\mathrm{km} \mathrm{s}^{-1}\right)$ & $50 \pm 10$ \\
$v \sin i\left(\mathrm{~km} \mathrm{~s}^{-1}\right)$ & $55 \pm 8$ \\
$\log (\mathrm{He} / \mathrm{H})+12$ & $11.0 \pm 0.20(10.99 \pm 0.01)$ \\
$\log (\mathrm{C} / \mathrm{H})+12$ & $8.0 \pm 0.15(8.33 \pm 0.04)$ \\
$\log (\mathrm{Mg} / \mathrm{H})+12$ & $7.6 \pm 0.10(7.56 \pm 0.05)$ \\
$\log (\mathrm{Si} / \mathrm{H})+12$ & $7.5 \pm 0.10(7.50 \pm 0.05)$ \\
\hline
\end{tabular}

Table 3. Changes in the heliocentric radial velocity of HD 93795.

\begin{tabular}{lc}
\hline Date & $v_{\mathrm{r}, \text { hel }}\left(\mathrm{km} \mathrm{s}^{-1}\right)$ \\
\hline 2014 March 9 & $-9.3 \pm 0.1$ \\
2018 December 21 & $-5.5 \pm 0.3$ \\
2019 February 5 & $-16.5 \pm 0.2$ \\
\hline
\end{tabular}

from 5 to $25 \mathrm{~km} \mathrm{~s}^{-1}$ in steps of $1 \mathrm{~km} \mathrm{~s}^{-1}$. Different chemical abundances and microturbulence velocities were simultaneously examined until finding the best match with the observations. The best-fitting model is displayed in Fig. 3 while the stellar parameters derived from this model are compiled in Table 2 Note that at the effective temperature of HD 93795, we could only constrain the chemical abundances of those elements with strong absorption lines whose transitions were included in our models, i.e. $\mathrm{Mg}, \mathrm{C}$ and $\mathrm{Si}$. The main lines used for the spectral modelling are marked in Fig. 3.

We used the IACOB-BRoAd code Simón-Díaz \& Herrero (2014) to measure the projected rotational $(v \sin i)$ and macroturbulence $\left(v_{\text {mac }}\right)$ velocities. This code characterizes the additional broadening in the line profiles combining Fourier transform and goodness-of-fit methodologies. The obtained velocities (see Table 2) are qualitatively in agreement with velocities measured by Simón-Díaz et al. (2017) in stars at the similar evolutionary stages (see their fig. 5).

Using the available three échelle spectra, we also measured the heliocentric radial velocity, $v_{\text {r,hel }}$, of HD 93795 in the way described in Gvaramadze et al. (2019b). The obtained values of $v_{\mathrm{r}, \text { hel }}$ (see Table 3 ) are similar to those measured by Humphreys $(1973,1978)$, who found radial velocity variations in the range between $\approx-4$ and $-21 \mathrm{kms}^{-1}$ (see Section2). The changes in $v_{\mathrm{r}, \text { hel }}$ suggest that HD 93795 might be a binary system, but they could also be caused by pulsations of the stellar photosphere (e.g. Jeffery \& Saio 2016). Time-series photometry and spectroscopy of HD 93795 are necessary to distinguish between these two possibilities.

Comparison of the three spectra also revealed changes in the Balmer line profiles, which are most prominent in the $\mathrm{H} \alpha$ line (see Fig.4). This line shows a distinctive P Cyg profile (suggesting that the star possesses a strong wind) and also has an emission component in the blue wing (note 


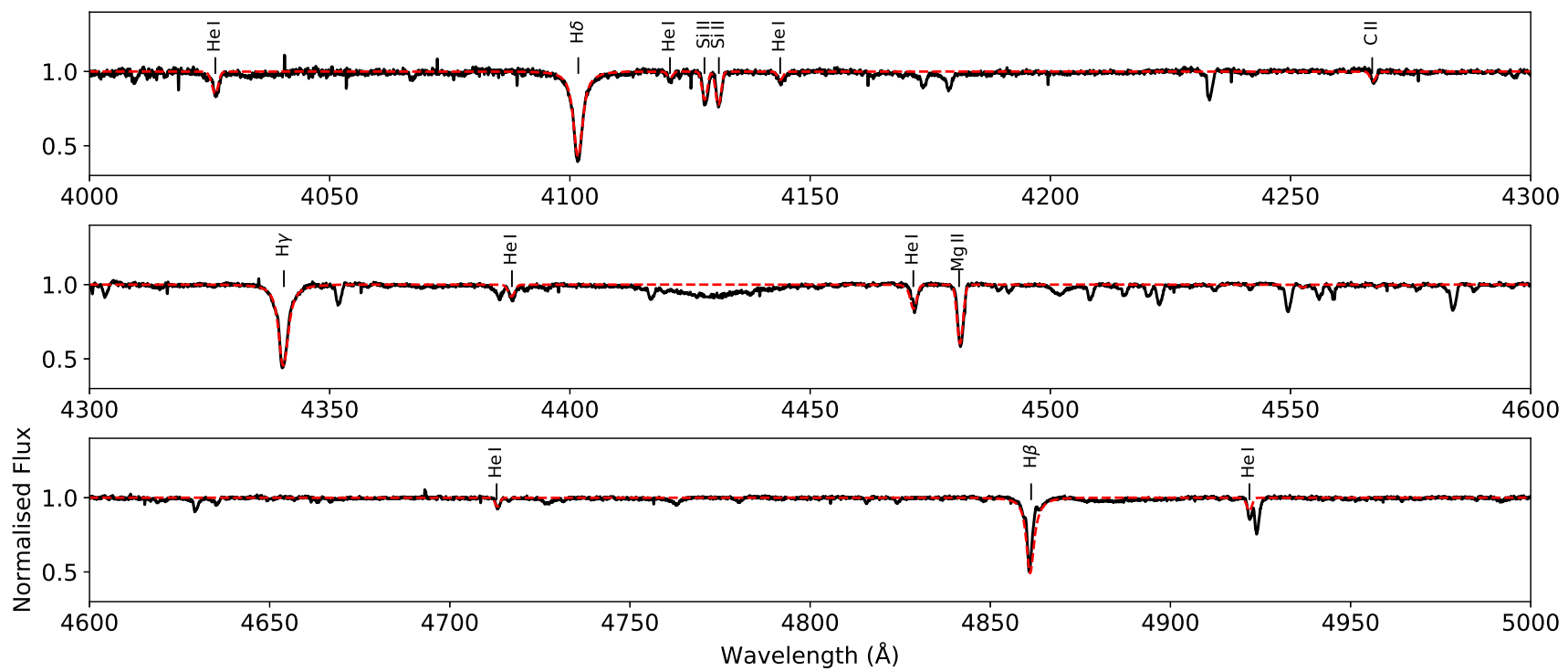

Figure 3. Parts of the normalized and rebinned spectrum of HD 93795 (black line), compared with the best-fitting FASTwIND model (red dashed line) with the parameters as given in Table 2 The lines fitted by the model are indicated.
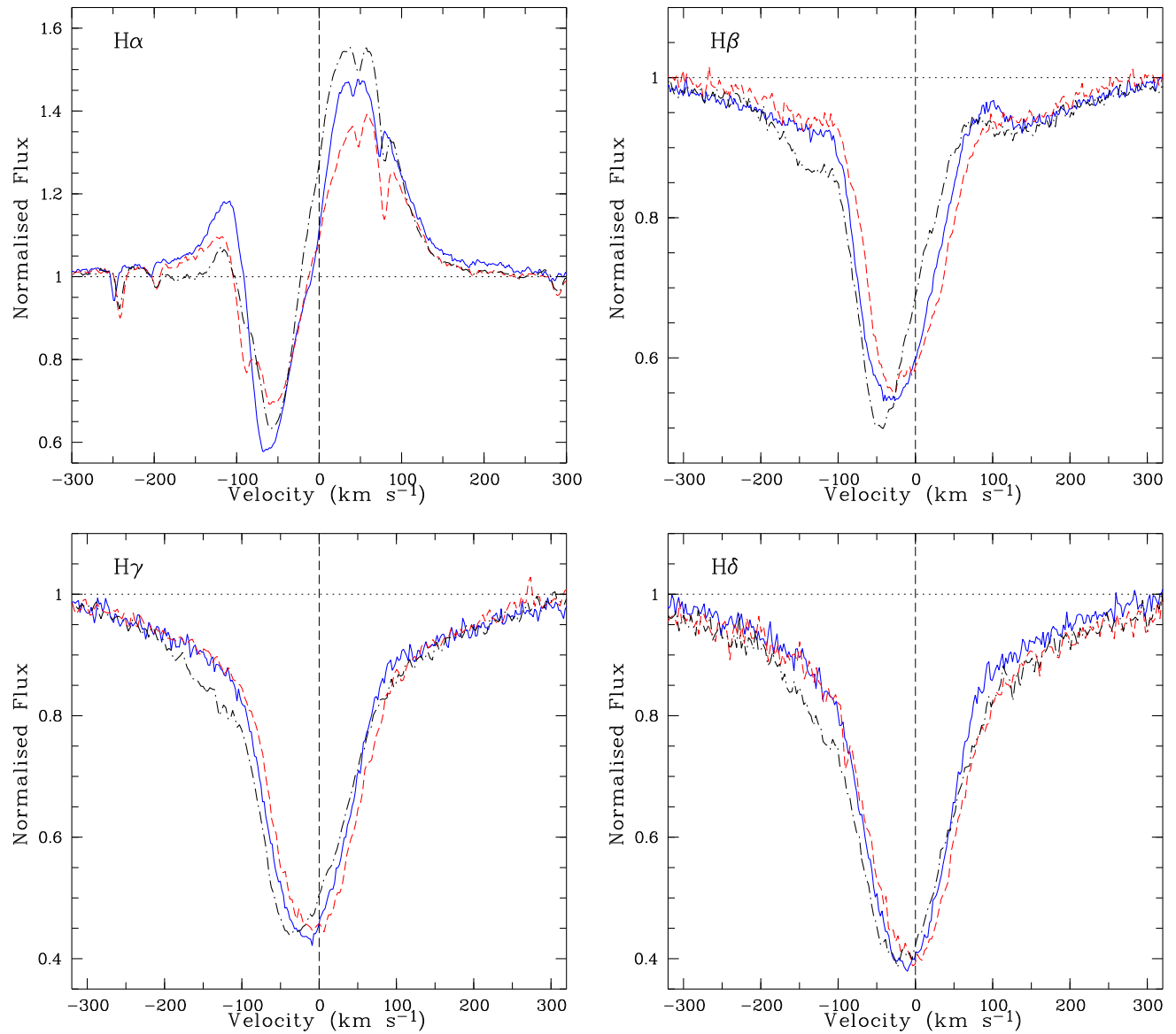

Figure 4. Changes in the Balmer line profiles in the spectra obtained with FEROS on 2014 March 9 (solid blue line) and HRS on 2018 December 21 (dashed red line) and 2019 February 5 (dot-dashed black line). Velocities are relative to the zero heliocentric radial velocity (vertical dashed line), corresponding to the rest wavelengths of the lines. Narrow dips in the $\mathrm{H} \alpha$ line profiles are due to telluric $\mathrm{H}_{2} \mathrm{O}$ absorption lines. 


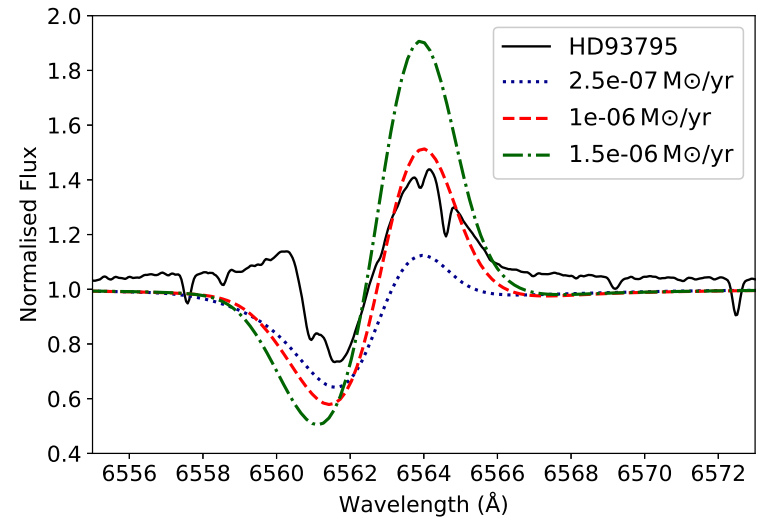

Figure 5. Comparison of the observed profile of the $\mathrm{H} \alpha$ line (from the 2019 spectrum) with synthetic profiles predicted by FASTWIND models with different values of the mass-loss rate as specified in the legend.

that narrow dips in the line profile are telluric in origin). The $\mathrm{H} \alpha$ line variability was detected in almost all late- $\mathrm{B}$ and early-A supergiants (e.g., Kaufer et al. 1996; Markova \& Valchev 2000; Verdugo et al. 2000), which in most cases could be characterised as a variable double-peaked emission pattern symmetric about the systemic velocity. By analogy with the $\mathrm{V} / \mathrm{R}$ variability in the $\mathrm{H} \alpha$ line profiles in Be stars, this variability was interpreted (e.g., Kaufer et al. 1996) in terms of an axially-symmetric stellar wind and/or a disc-like structure around the star.

In Fig. 5. we compare the observed $\mathrm{H} \alpha$ line profile from the 2019 spectrum with the FASTWIND synthetic profiles obtained for three different mass-loss rates $\dot{M}$ (indicated in the legend of the figure). To produce the synthetic profiles, we adopted a terminal wind velocity of $v_{\infty}=220 \mathrm{~km} \mathrm{~s}^{-1}$, based on the effective temperature of HD 93795 and the empirical calibration in Kudritzki \& Puls (2000), and a wind velocity law exponent $\beta=3.5$ (see equation (3) in Castro et al. 2012). No clumping was included in the models.

Fig. 5 shows that although the intensity of the observed line matches a mass-loss rate of $\approx 10^{-6} \mathrm{M}_{\odot} \mathrm{yr}^{-1}$, the synthetic profiles did not reproduce the wings of the line. This discrepancy could be due to shortcomings of our models or/and due to non-spherical geometry of the stellar wind (e.g. Kaufer et al. 1996; Kudritzki et al. 1999).

\subsection{Reddening, luminosity and evolutionary status of HD 93795}

To determine the $V$-band reddening towards HD 93795, one can use the photometric data from Table1 and the intrinsic colours $(B-V)_{0}$ and $(J-K)_{0}$ of B9 supergiants of, respectively, $-0.06 \mathrm{mag}$ (Firnstein \& Przybilla 2012) and -0.05 mag (Wenger 2014):

$A_{V}=R_{V}\left[(B-V)-(B-V)_{0}\right]$

and

$A_{V}=5.89\left[(J-K)-(J-K)_{0}\right]$,

where $R_{V}=A_{V} / E(B-V)$ is total-to-selective absorption ratio, $K=K_{\mathrm{s}}+0.04 \mathrm{mag}$ (Carpenter 2001). To derive equation (2), we used the extinction law of Rieke \& Lebofsky (1985). From equations (1) and (2), one finds $A_{V}=0.86 R_{V}$ mag and $A_{V}=3.18 \mathrm{mag}$, respectively, which would agree with each other if $R_{V} \approx 3.7$. This suggests that the reddening towards HD 93795 is anomalous, which is in good agreement with the results by Herbst (1976) and Forte (1978), showing that the extinction law towards the Carina Nebula is characterized by significantly larger values of $R_{V}(\approx 4-5)$ then the "normal" value of 3.1.

Adopting $A_{V}=3.18 \mathrm{mag}$ and using the bolometric correction for $\mathrm{B} 9$ supergiants of $\mathrm{BC}=-0.31 \mathrm{mag}$ (Firnstein \& Przybilla 2012), one finds the absolute $V$-band magnitude and luminosity of HD 93795 of

$M_{V}=V-5 \log (d / \mathrm{pc})+5-A_{V} \approx-8.17_{-0.40}^{+0.48} \mathrm{mag}$

and

$\log \left(L / \mathrm{L}_{\odot}\right)=0.4\left(4.74-\mathrm{M}_{\mathrm{V}}-\mathrm{BC}\right) \approx 5.3 \pm 0.2$,

respectively. For the error calculation, only uncertainties in the adopted distance of $5 \pm 1 \mathrm{kpc}$ were considered. The obtained luminosity implies that the zero-age main-sequence mass of HD 93795 was $\approx 25 \pm 5 \mathrm{M}_{\odot}$ (e.g. Ekström et al. 2012 ). In the case if the star is located at $d=2.3 \mathrm{kpc}$, its luminosity and initial mass would be equal to $\log \left(L / \mathrm{L}_{\odot}\right) \approx 4.6$ and $\approx 15 \mathrm{M}_{\odot}$, respectively.

The luminosity of HD 93795 could also be estimated using the flux-weighted gravity-luminosity relationship $(\mathrm{Ku}-$ dritzki, Bresolin \& Przybilla 2003):

$M_{\mathrm{bol}}=(3.41 \pm 0.16)\left(\log g_{\mathrm{F}}-1.5\right)-(8.02 \pm 0.04)$,

where $M_{\mathrm{bol}}=M_{V}+\mathrm{BC}$ is the bolometric magnitude and $\log g_{\mathrm{F}}=\log g-4 \log \left(T_{\text {eff }} \times 10^{-4}\right)$. For $\log g=1.6 \pm 0.1$ and $T_{\text {eff }}=11 \pm 1 \mathrm{kK}$, one finds from equation (3) that $M_{\mathrm{bol}}=$ $-8.3 \pm 1.0 \mathrm{mag}$, which corresponds to the stellar luminosity of $\log \left(L / \mathrm{L}_{\odot}\right)=5.2 \pm 0.4$. Taken at face value, this estimate agrees well with that based on the Gaia distance, but still did not exclude the possibility that HD 93795 is located at a shorter distance.

Our spectral modelling showed that the helium abundance in HD 93795 is close to the CAS value, while the carbon abundance is only slightly depleted (see Table2). This indicates that HD 93795 only recently left the main sequence and is evolving redward (to cooler temperatures) for the first time. Correspondingly, the luminosity and the temperature of HD 93795 would imply the age of this star of $\gtrsim 10 \mathrm{Myr}$ (Ekström et al. 2012). This age is much older than the age of the central massive star clusters in the Carina Nebula of 1-3 Myr (Hur et al. 2012), but comparable to the kinematic age of the Car OB1 association of 10 Myr (Melnik \& Dambis 2017).

\section{RSS SPECTROSCOPY OF THE NEBULA}

Figure 6] shows the $\mathrm{H} \alpha$ and [N II] $\lambda 6584$ emission line intensity profiles along the RSS slit, oriented along the presumed axis of rotational symmetry of the nebula $\left(\mathrm{PA}=80^{\circ}\right)$. The figure shows that HD 93795 is surrounded by a region of strong nitrogen emission, extended between $\approx+20$ arcsec and -15 arcsec, i.e. within the angular extent of the IR nebula. We will refer to this region as the "nebula". There 


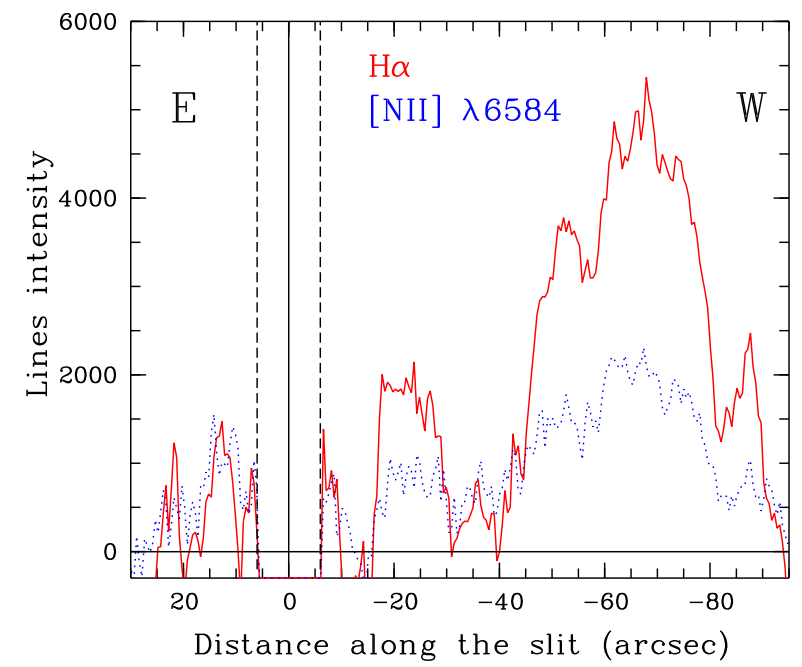

Figure 6. Variations of the $\mathrm{H} \alpha$ and $[\mathrm{N}$ II] $\lambda 6584$ emission line intensities along the slit with $\mathrm{PA}=80^{\circ}$, shown, respectively, with a solid (red) and dashed (blue) lines. The solid vertical line corresponds to the position of HD 93795, while the dashed vertical lines at \pm 6 arcsec from the solid line mark the area where no nebular emission was detected because of the effect of HD 93795. $\mathrm{E}-\mathrm{W}$ direction of the slit is shown.

is also another region of strong nitrogen emission to the west of the star between $\approx-30$ and -40 arcsec. Interestingly, this region corresponds to a knot of enhanced IR emission, most clearly visible in the IRAC $8 \mu \mathrm{m}$ image (see Fig.11). We will refer to this region as the "knot" and discuss it in Section 6 Besides, there are also two regions of bright $\mathrm{H} \alpha$ emission to the west of the star, located between $\approx-18$ and -30 arcsec and $\approx-40$ and -80 arcsec; hereafter, respectively, "region A" and "region B".

In Fig. 7 we present spectra of the above four regions. All of them show Balmer emission lines and two strong forbidden emission doublets of [N II] $\lambda \lambda 6548,6583$ and [S II] $\lambda \lambda 6716,6731$. The spectrum of the region $\mathrm{B}$ also clearly shows the presence of the [O III] $\lambda 5007$ emission lines. In Table 4 we give for each region the observed intensities of the detected lines normalized to $\mathrm{H} \beta, \mathrm{F}(\lambda) / \mathrm{F}(\mathrm{H} \beta)$, the reddening-corrected line intensity ratios, $\mathrm{I}(\lambda) / \mathrm{I}(\mathrm{H} \beta)$, the logarithmic extinction coefficient, $C(\mathrm{H} \beta)$, the colour excess $E(B-V)$, and the electron number density $n_{\mathrm{e}}([\mathrm{S} \mathrm{II}])$ derived from the $[\mathrm{S} I I] \lambda \lambda 6716,6731$ line ratio. The lines in Table 4 were measured with program described in Kniazev et al. (2004).

One can see that the [S II] doublet is particularly strong in the spectra of the nebula and the knot, which suggests that their emission is the result of shock excitation. Indeed, using the $\log (\mathrm{H} \alpha /[\mathrm{N} \mathrm{II}])$ versus $\log (\mathrm{H} \alpha /[\mathrm{S} \mathrm{II}])$ diagnostic diagram (e.g. Sabbadin, Minello \& Bianchini 1977; Kniazev, Pustilnik \& Zucker 2008), one finds that the nebula and the knot are located in the same area as supernova remnants, whose optical emission is due to shock heating. Similarly, the line ratios in regions $\mathrm{A}$ and $\mathrm{B}$ place them in the area occupied by $\mathrm{H}$ II regions.

Note that the colour excess towards the nebula is lower by $\approx 0.5$ mag than that derived for HD 93795 (see Section 4.3. We attribute this difference to internal extinc- tion in the ridge, which we interpret as a disc-like structure viewed edge-on (see next section). On the other hand, the colour excesses measured for all four regions along the slit are consistent with each other within the margins of errors, and are within the range of colour excesses found for stars in the Carina Nebula (e.g. Herbst 1976; Forte 1978). This could be considered as an indication that HD 93795 is located in the Carina Nebula as well.

\section{DISCUSSION}

The results of our spectral analysis indicate that HD 93795 is one more example of a massive star which managed to produce a compact circumstellar nebula soon after it left the main sequence. We interpret the bright ridge within the nebula as a flattened (disc-like) structure viewed edge-on. If correct, this interpretation would suggest that rotation have played a role in shaping the nebula. If HD 93795 was born as a single star, then one needs to assume that it was, at least at the time of formation of the nebula, a very fast rotator. In this case, the axial symmetry axis of the nebula is defined by the rotational axis of the star. Moreover, the measured low projected rotational velocity of HD 93795 would be equal to the true rotational velocity, meaning that the star has significantly spun down since the formation of the nebula. Alternatively, HD 93795 might be a product of binary evolution, and in this case the symmetry axis of the nebula would coincide with the angular momentum vector of the binary orbit. Let us discuss possible scenarios for the origin of axiallysymmetric nebulae around relatively unevolved single and binary stars.

In the early stages of a single massive star's evolution the formation of an axially-symmetric circumstellar nebula could be related to the bi-stability jump (Pauldrach \& Puls 1990; Lamers \& Pauldrach 1991), which is manifested in drastic changes in the wind properties caused by decrease of the stellar effective temperature below some critical value ( $\approx 21000-25000 \mathrm{~K}$; Vink, de Koter \& Lamers 1999). Recent bi-stability models by Vink (2018) predict that decrease of $T_{\text {eff }}$ from $\approx 25000$ to $17000 \mathrm{~K}$ is accompanied by about an order of magnitude increase in $\dot{M}$ and by about the same magnitude decrease in $v_{\infty}$, leading to a factor of $\sim 100$ increase in the density of the stellar wind. In fast-rotating stars $T_{\text {eff }}$ is lower near the stellar equator because of the effect of gravity-darkening. In these stars the bi-stability jump first takes place at low latitudes, which might result in a dense axially-symmetric equatorial outflow (Lamers \& Pauldrach 1991; Lamers, Snow \& Lindholm 1995).

The main problem with this scenario is that the size of nebulae created by the rotationally induced bi-stability jump mechanism should be much larger than the size of the nebula around HD 93795. Indeed, the stellar evolutionary models (e.g. Ekström et al. 2012) show that in fast-rotating stars with the initial mass in the range derived for HD 93795 the effective temperature decreases from 25000 to $17000 \mathrm{~K}$ on the time scale from several 1000 to $\approx 10000 \mathrm{yr}$. During this time, the equatorial wind will spread over a distance from several to about $10 \mathrm{pc}$, which is an order of magnitude larger than the radius of the nebula around HD 93795 of $\approx 0.36(d / 5 \mathrm{kpc}) \mathrm{pc}$. In this connection, we note that the radii of equatorial rings of all five previously known bipolar 

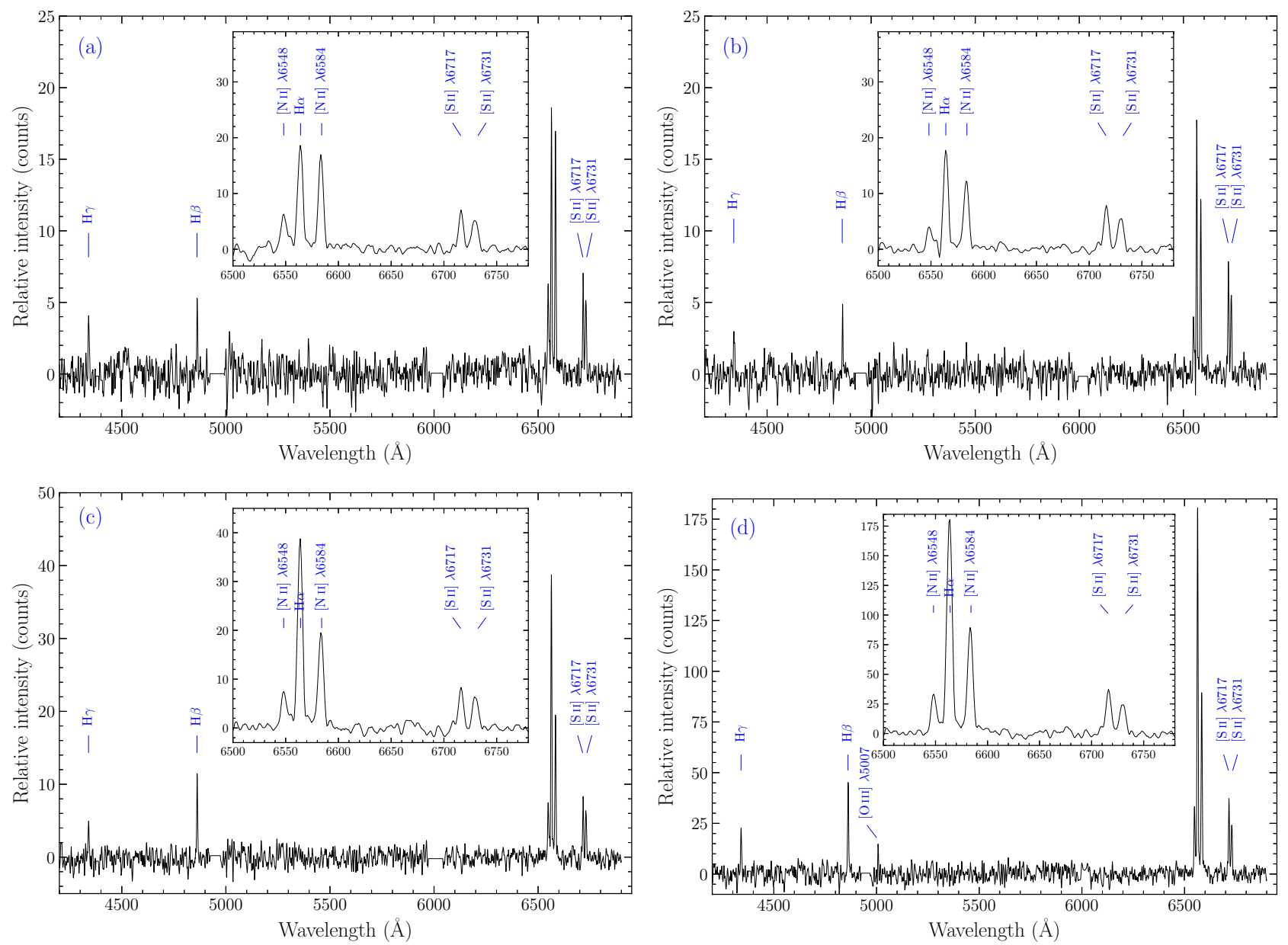

Figure 7. 1D RSS spectra of the nebula (panel a), knot (panel b) and regions A and B (panels c and d, respectively). The major emission lines are marked. The inserts show parts of the spectra around the $\mathrm{H} \alpha$ line. The gaps in the spectra at $\approx 4900$ and $6000 \AA$ are due to gaps between CCDs. See text for details.

Table 4. Line intensities of the circumstellar nebula and three other regions along the RSS slit. See text for details.

\begin{tabular}{|c|c|c|c|c|c|c|c|c|}
\hline \multirow[b]{2}{*}{$\lambda_{0}(\AA)$ Ion } & \multicolumn{2}{|c|}{ Nebula } & \multicolumn{2}{|c|}{ Knot } & \multicolumn{2}{|c|}{ Region A } & \multicolumn{2}{|c|}{ Region B } \\
\hline & $\mathrm{F}(\lambda) / \mathrm{F}(\mathrm{H} \beta)$ & $\mathrm{I}(\lambda) / \mathrm{I}(\mathrm{H} \beta)$ & $\mathrm{F}(\lambda) / \mathrm{F}(\mathrm{H} \beta)$ & $\mathrm{I}(\lambda) / \mathrm{I}(\mathrm{H} \beta)$ & $\mathrm{F}(\lambda) / \mathrm{F}(\mathrm{H} \beta)$ & $\mathrm{I}(\lambda) / \mathrm{I}(\mathrm{H} \beta)$ & $\mathrm{F}(\lambda) / \mathrm{F}(\mathrm{H} \beta)$ & $\mathrm{I}(\lambda) / \mathrm{I}(\mathrm{H} \beta)$ \\
\hline $4340 \mathrm{H} \gamma$ & $0.63 \pm 0.13$ & $0.75 \pm 0.15$ & $0.50 \pm 0.14$ & $0.60 \pm 0.17$ & $0.47 \pm 0.05$ & $0.52 \pm 0.06$ & $0.45 \pm 0.04$ & $0.53 \pm 0.04$ \\
\hline $4861 \mathrm{H} \beta$ & $1.00 \pm 0.12$ & $1.00 \pm 0.12$ & $1.00 \pm 0.13$ & $1.00 \pm 0.13$ & $1.00 \pm 0.07$ & $1.00 \pm 0.07$ & $1.00 \pm 0.06$ & $1.00 \pm 0.06$ \\
\hline 5007 [O III] & - & - & - & - & - & - & $0.22 \pm 0.04$ & $0.21 \pm 0.04$ \\
\hline $6548[\mathrm{~N} \mathrm{II}]$ & $1.35 \pm 0.14$ & $0.90 \pm 0.10$ & $1.02 \pm 0.13$ & $0.64 \pm 0.09$ & $0.71 \pm 0.06$ & $0.55 \pm 0.05$ & $0.77 \pm 0.04$ & $0.52 \pm 0.03$ \\
\hline $6563 \mathrm{H} \alpha$ & $4.38 \pm 0.38$ & $2.89 \pm 0.28$ & $4.61 \pm 0.45$ & $2.89 \pm 0.31$ & $3.76 \pm 0.21$ & $2.89 \pm 0.18$ & $4.30 \pm 0.19$ & $2.89 \pm 0.14$ \\
\hline 6584 [N II] & $4.17 \pm 0.35$ & $2.74 \pm 0.26$ & $3.18 \pm 0.31$ & $1.99 \pm 0.21$ & $2.16 \pm 0.12$ & $1.66 \pm 0.10$ & $2.35 \pm 0.10$ & $1.57 \pm 0.08$ \\
\hline 6717 [S II] & $1.56 \pm 0.17$ & $1.00 \pm 0.12$ & $2.11 \pm 0.23$ & $1.28 \pm 0.15$ & $0.79 \pm 0.07$ & $0.60 \pm 0.05$ & $0.89 \pm 0.05$ & $0.58 \pm 0.04$ \\
\hline 6731 [S II] & $1.39 \pm 0.15$ & $0.88 \pm 0.11$ & $1.64 \pm 0.19$ & $0.99 \pm 0.12$ & $0.67 \pm 0.06$ & $0.50 \pm 0.05$ & $0.64 \pm 0.05$ & $0.41 \pm 0.03$ \\
\hline$C(\mathrm{H} \beta)$ & \multicolumn{2}{|c|}{$0.55 \pm 0.11$} & \multicolumn{2}{|c|}{$0.61 \pm 0.12$} & \multicolumn{2}{|c|}{$0.34 \pm 0.07$} & \multicolumn{2}{|c|}{$0.52 \pm 0.06$} \\
\hline$E(B-V)$ & \multirow{2}{*}{\multicolumn{2}{|c|}{$\begin{array}{c}0.37 \pm 0.07 \\
340^{+420} \mathrm{~cm}^{-3}\end{array}$}} & \multirow{2}{*}{\multicolumn{2}{|c|}{$\begin{array}{c}0.41 \pm 0.08 \\
130_{-120}^{+325} \mathrm{~cm}^{-3}\end{array}$}} & \multirow{2}{*}{\multicolumn{2}{|c|}{$\begin{array}{c}0.23 \pm 0.05 \\
250_{-175}^{+265} \mathrm{~cm}^{-3}\end{array}$}} & \multirow{2}{*}{\multicolumn{2}{|c|}{$\begin{array}{c}0.35 \pm 0.04 \\
25_{-15}^{+135} \mathrm{~cm}^{-3}\end{array}$}} \\
\hline$n_{\mathrm{e}}([\mathrm{Si} \mathrm{II}])$ & & & & & & & & \\
\hline
\end{tabular}

nebulae produced by blue supergiants (see table 7 in Gvaramadze et al. 2015 for main properties of these stars and their circumstellar rings) span a narrow range from $\approx 0.2$ to 0.3 pc, and that their expansion velocities of $\approx 10-30 \mathrm{~km} \mathrm{~s}^{-1}$ are about one or two orders of magnitude lower than the wind velocity of blue supergiants on the cool side of the bi- stability jump. This strongly suggests that the bi-stability jump mechanism cannot be responsible for the origin of these nebulae. Although the expansion velocity of the nebula around HD 93795 is unknown, its small size also suggests that the bi-stability jump is not responsible for its origin. We therefore conclude that compact axially-symmetric nebulae 
hardly can be produced by single massive stars during their early post-main-sequence evolution.

Among the five already known compact axiallysymmetric nebulae (rings) produced by blue supergiants, the most studied one is the equatorial ring around $\mathrm{Sk}-69^{\circ} 202$ (Wampler et al. 1990; Jakobsen et al. 1991) — the progenitor star of SN 1987A. The proposed models for its origin converge on the idea that $\mathrm{Sk}-69^{\circ} 202$ was a binary system that merged shortly $(\sim 10000 \mathrm{yr})$ before the supernova explosion (e.g. Podsiadlowski 1992; Collins et al. 1999; Morris \& Podsiadlowski 2007) to produce a highly flattened circumstellar structure slowly expanding in the system's orbital plane. Presumably, this structure is composed either of material expelled by the merging binary during the common-envelope phase (Morris 1981) or of wind material lost by the inflated merger product during its contraction into a blue supergiant (Heger \& Langer 1998).

The photospheric abundances of other four blue supergiants (Sher 25, HD 168625, [SBW2007] 1 and MN18) with equatorial rings indicate that these stars did not evolve through the red supergiant phase (e.g. Smartt et al. 2002; Smith et al. 2007; Gvaramadze et al. 2015), meaning that the rings were produced while the stars were in the main sequence phase or at the beginning of the blue supergiant phase (cf. Lamers et al. 2001). The luminosities of these stars range from $\log \left(L / \mathrm{L}_{\odot}\right) \approx 4.7$ to 5.8 , which indicates that the stellar luminosity (or initial mass of the star) is not a crucial factor in formation of circumstellar nebulae. The effective temperatures of these stars also span quite a large range from $\approx 11000$ to $22000 \mathrm{~K}$ (spectral types from B6 to $\mathrm{B} 1)$. What units them is the similarity of the parameters (size, expansion velocity, number density) of their circumstellar rings (see table 7 in Gvaramadze et al. 2015). This points to the possibility that perhaps there is a universal mechanism for formation of rings around massive stars.

Since most of massive stars are formed in binary and multiple systems (e.g. Sana et al. 2012; Chini et al. 2012) and because massive binaries can merge both on the main sequence and in later stages of their evolution (e.g. de Mink et al. 2014), we suggest that the origin of the nebulae around HD 93795 and the four other blue supergiants evolving redwards for the first time is also due to merger of binary systems. This implies that the equatorial rings should be shortlived objects because after thermal relaxation of the merger products the fast wind from the newly formed blue supergiants quickly disperse them. Assuming that all mergers result in the origin of axially-symmetric nebulae and that the lifetime of these nebulae is $\sim 10,000 \mathrm{yr}$, one can show (see Gvaramadze et al. 2015) that there are should be $\sim 10$ such nebulae in the Milky Way.

During the initial stage of interaction between the fast wind and the slow dense material of the equatorial ejecta, the stellar wind will flow freely in the polar directions to create bipolar lobes. The "horns" on each side of the ridge in the nebula around HD 93795 may represent the beginning of these lobes, while the strong nitrogen emission detected close to the star may come from the equatorial ejecta shocked by the stellar wind. If so, then the knot of shocked gas to the west of HD 93795 may be a cloudlet in the interstellar medium, which was accidentally caught in the path of the stellar wind flowing in the polar direction.

Finally, we discuss the possible membership of
HD 93795 of the Car OB1 association. While taken at face value the Gaia parallax indicates that HD 93795 is a factor of two more distant than Car OB1, the distance to HD 93795 could be overestimated because of the systematic offset in the Gaia DR2 parallaxes. The large distance to HD 93795 is also suggested by the flux-weighted gravity-luminosity relationship for blue supergiants, but the uncertainty inherent to this relationship does not exclude the possibility that the star is at a smaller distance. On the other hand, the colour excess towards the region of shocked gas in the close vicinity of HD 93795 (we associate this material with the slow equatorial ejecta shocked by the fast stellar wind) is typical of stars in the Carina Nebula, which points to the possibility that HD 93795 is located in the Carina Nebula as well. Although the existing data did not allow us to unambiguously constrain the distance to HD 93795, we conclude that this star could be a member of the Car OB1 association. Hopefully, the forthcoming third Gaia data release will resolve this issue.

\section{SUMMARY}

We have discovered a compact IR nebula around the emission-line star HD 93795 using archival data from the Spitzer Space Telescope. Follow-up optical spectroscopy of HD 93795 with the Southern African Large Telescope led to its classification as a B9 Ia star, while modelling of its spectrum showed that HD 93795 is a relatively unevolved star moving redward for the first time. These findings support the previous view that massive stars can produce circumstellar nebulae while they are still on the main sequence or shortly after they left it.

The IR nebula has a square shape with a bright ridge in the middle and two symmetric flanks perpendicular to the ridge. We interpret the ridge as an axially-symmetric flattened structure viewed edge-on, and suggest that the flanks outline the beginning of bipolar lobes. The axiallysymmetric shape of the nebula suggests that HD 93795 was either a single fast-rotating star or a close binary system, which has merged in the recent past. In the first case, the only possible way to produce an axially-symmetric nebula is through the rotationally induced bi-stability jump mechanism. The size and expansion velocity of such a nebula, however, would be much larger than those of the known circumstellar rings around blue supergiants. We therefore conclude, that the merger of binary systems appears to be a more natural process leading to the formation of compact slowly expanding and flattened circumstellar nebulae.

\section{ACKNOWLEDGEMENTS}

This work is based on observations obtained with the Southern African Large Telescope (SALT), programmes 2013-1-RSA_OTH-014， 2015-1-SCI-017， 2018-1-MLT-008, and collected at the European Southern Observatory under ESO programme 092.A-9020(A). V.V.G. acknowledges support from the Russian Science Foundation under grant 19-12-00383. A.Y.K. acknowledges support from the National Research Foundation (NRF) of South Africa. This research has made use of the NASA/IPAC Infrared Science 
Archive, which is operated by the Jet Propulsion Laboratory, California Institute of Technology, under contract with the National Aeronautics and Space Administration, the SIMBAD data base and the VizieR catalogue access tool, both operated at CDS, Strasbourg, France, and data from the European Space Agency (ESA) mission Gaia (https://www.cosmos.esa.int/gaia), processed by the Gaia Data Processing and Analysis Consortium (DPAC, https://www.cosmos.esa.int/web/gaia/dpac/consortium).

Funding for the DPAC has been provided by national institutions, in particular the institutions participating in the Gaia Multilateral Agreement.

\section{REFERENCES}

Azzopardi M., 1987, A\&AS, 69, 421

Baldwin J. A., Stone R. P. S., 1984, MNRAS, 206, 241

Barnes S. I. et al., 2008, in McLean I. S., Casali M. M., eds, Proc. SPIE Conf. Ser. Vol. 7014, Ground-based and Airborne Instrumentation for Astronomy II. SPIE, Bellingham, p. $70140 \mathrm{~K}$

Bramall D. G. et al., 2010, in McLean I. S., Ramsay S. K., Takami H., eds, Proc. SPIE Conf. Ser. Vol. 7735, Ground-based and Airborne Instrumentation for Astronomy III. SPIE, Bellingham, p. $77354 \mathrm{~F}$

Bramall D. G. et al., 2012, in McLean I. S., Ramsay S. K., Takami H., eds, Proc. SPIE Conf. Ser. Vol. 8446, Ground-based and Airborne Instrumentation for Astronomy IV. SPIE, Bellingham, p. $84460 \mathrm{~A}$

Buckley D. A. H., Swart G. P., Meiring J. G., 2006, Proc. SPIE, 6267,32

Burgh E. B., Nordsieck K. H., Kobulnicky H. A., Williams T. B., O'Donoghue D., Smith M. P., Percival J. W., 2003, Proc. SPIE, 4841, 1463

Carpenter J. M., 2001, AJ, 121, 2851

Castro N. et al., 2012, A\&A, 542, A79

Collins T. J. B., Frank A., Bjorkman J. E., Livio M., 1999, ApJ, 512,322

Crause L. A. et al., 2014, in Ramsay S. K., McLean I. S., Takami H., eds, Proc. SPIE Conf. Ser. Vol. 9147, Ground-based and Airborne Instrumentation for Astronomy V. SPIE, Bellingham, p. 91476T

Crawford S. M. et al., 2010, in Silva D. R., Peck A. B., Soifer B. T., eds, Proc. SPIE Conf. Ser. Vol. 7737, Observatory Operations: Strategies, Processes, and Systems III. SPIE, Bellingham, p. 773725

Cutri R. M. et al., 2003, VizieR Online Data Catalog, 2246, 0

Drew J. E. et al., 2014, MNRAS, 440, 2036

Egret D., Didelon P., McLean B. J., Russell J. L., Turon C., 1992, A\&A, 258, 217

Ekström S. et al., 2012, A\&A, 537, A146

Evans C. J., Howarth I. D., Irwin M. J., Burnley A. W., Harries T. J., 2004, MNRAS, 353, 601

Fazio G. G. et al., 2004, ApJS, 154, 10

Feigelson E. D. et al., 2011, ApJS, 194, 9

Firnstein M., Przybilla N., 2012, A\&A 543, A80

Forte J. C., 1978, AJ, 83, 1199

Gaia Collaboration, Brown A. G. A., Vallenari A., Prusti T., de Bruijne J. H. J., Babusiaux C., Bailer-Jones C. A. L., 2018, A\&A, 616, A1

Gvaramadze V. V., Bomans D. J., 2008, A\&A, 490, 1071

Gvaramadze V. V., Kniazev A. Y., 2017, in Miroshnichenko A.S., Zharikov S.V., Korcakova D., Wolf M., eds, ASP Conf. Ser. Vol. 508, The B[e] Phenomenon. Forty Years of Studies. Astron. Soc. Pac., San Francisco, p. 207
Gvaramadze V. V., Kniazev A. Y., Fabrika S., 2010, MNRAS, 405,1047

Gvaramadze V. V. et al., 2012, MNRAS, 421, 3325

Gvaramadze V. V., Miroshnichenko A. S., Castro N., Langer N., Zharikov S. V., 2014, MNRAS, 437, 2761

Gvaramadze V. V. et al., 2015, MNRAS, 454, 219

Gvaramadze V. V., Kniazev A. Y., Castro N., Grebel E. K., 2019a, AJ, 157, 53

Gvaramadze V. V., Maryeva O. V., Kniazev A. Y., Alexashov D. B., Castro N., Langer N., Katkov I. Y., 2019b, MNRAS, 482, 4408

Hamuy M., Walker A. R., Suntzeff N. B., Gigoux P., Heathcote S. R., Phillips M. M., 1992, PASP, 104, 533

Hamuy M., Suntzeff N. B., Heathcote S. R., Walker A. R., Gigoux P., Phillips M. M., 1994, PASP, 106, 566

Heger A., Langer N., 1998, A\&A, 334, 210

Heiles C., 2000, AJ, 119, 923

Hendry M. A., Smartt S. J., Skillman E. D., Evans C. J., Trundle C., Lennon D. J., Crowther P. A., Hunter I., 2008, MNRAS, 388,1127

Herbst W., 1976, ApJ, 208, 923

Hoffleit D., 1956, ApJ, 124, 61

Houk N., Cowley A. P., 1975, Catalogue of Two-dimensional Spectral Types for the HD Stars, Vol. 1. Univ. Michigan, Ann Arbor.

Humphreys R. M., 1973, A\&AS, 9, 85

Humphreys R. M., 1978, ApJS, 38, 309

Hur H., Sung H., Bessell M. S., 2012, AJ, 143, 41

Jakobsen P. et al., 1991, ApJ, 369, L63

Jeffery C. S., Saio H., 2016, MNRAS, 458, 1352

Kaufer A., Stahl O., Wolf B., Gaeng T., Gummersbach C. A., Kovacs J., Mandel H., Szeifert T., 1996, A\&A, 305, 887

Keenan P. C., Hynek J. A., 1950, ApJ, 111, 1

Kniazev A., 2017, The list of spectrophotometric standard stars for HRS, p. 1; https://astronomers.salt.ac.za/software/hrspipeline/

Kniazev A. Y., Gvaramadze V. V., 2015, Proceedings of the SALT Science Conference 2015 (SSC2015). Stellenbosch Institute of Advanced Study, South Africa, p. 49

Kniazev A. Y., Pustilnik S. A., Grebel E. K., Lee H., Pramskij A. G., 2004, ApJS, 153, 429

Kniazev A. Y., Pustilnik S. A., Zucker D. B., 2008, MNRAS, 384, 1045

Kniazev A. Y., Gvaramadze V. V., Berdnikov L. N., 2016, MNRAS, 459, 3068

Kniazev A. Y. et al., 2008, MNRAS, 388, 1667

Kniazev A. Y. Usenko I. A., Kovtyukh V. V., Berdnikov L. N., 2019, Astrophys. Bull., 74, 208

Kobulnicky H. A., Nordsieck K. H., Burgh E. B., Smith M. P., Percival J. W., Williams T. B., ODonoghue D., 2003, Proc. SPIE, 4841, 1634

Kudritzki R.-P., Puls J., 2000, ARA\&A, 38, 613

Kudritzki R. P., Bresolin F., Przybilla N., 2003, ApJ, 582, L83

Kudritzki R. P., Puls J., Lennon D. J., Venn K. A., Reetz J., Najarro F., McCarthy J. K., Herrero A., 1999, A\&A, 350, 970

Lamers H. J. G. L. M., Pauldrach A. W. A., 1991, A\&A, 244, L5

Lamers H. J. G. L. M., Snow T. P., Lindholm D. M., 1995, ApJ, 455,269

Lamers H. J. G. L. M., Nota A., Panagia N., Smith L. J., Langer N., 2001, ApJ, 551, 764

Lim B., Nazé Y., Gosset E., Rauw G., 2019, MNRAS, 490, 440

Lindegren L. et al., 2018, A\&A, 616, A2

Mahy L., Hutsemékers D., Royer P., Waelkens C., 2016, A\&A, 594, A94

MacConnell D. J., 1981, A\&AS, 44, 387

Markova N., Valchev T., 2000, A\&A, 363, 995

Mel'nik, A. M., \& Dambis, A. K. 2017, MNRAS, 472, 3887 
Morris M., 1981, ApJ, 249, 572

Morris T., Podsiadlowski P., 2007, Science, 315, 1103

Nieva M.-F., Przybilla N., 2012, A\&A, 539, A143

O'Donoghue D. et al., 2006, MNRAS, 372, 151

Parker Q. A. et al., 2005, MNRAS, 362, 689

Pauldrach A. W. A., Puls J., 1990. A\&A, 237. 409

Podsiadlowski P., 1992, PASP, 104, 717

Puls J., Urbaneja M. A., Venero R., Repolust T., Springmann U., Jokuthy A., Mokiem M. R., 2005, A\&A, 435, 669

Rieke G. H., Lebofsky M. J., 1985, ApJ, 288, 618

Rieke G. H. et al., 2004, ApJS, 154, 25

Rivero González J. G., Puls J., Massey P., Najarro F., 2012, A\&A, 543, A95

Sabbadin F., Minello S., Bianchini A., 1977, A\&A, 60, 147

Santolaya-Rey A. E., Puls J., Herrero A., 1997, A\&A, 323, 488

Simón-Díaz S., Herrero A., 2014, A\&A, 562, A135

Simón-Díaz S., Godart M., Castro N., Herrero A., Aerts C., Puls J., Telting J., Grassitelli L., 2017, A\&A, 597, A22

Skrutskie M. F. et al., 2006, AJ, 131, 1163

Smartt S. J., Lennon D. J., Kudritzki R. P., Rosales F., Ryans R. S. I., Wright N., 2002, A\&A, 391, 979

Smith N., Bally J., Walawender J., 2007, AJ, 134, 846

Stassun K. G., Torres G., 2018, ApJ, 862, 61

Stephenson C. B., Sanduleak N., 1971, Publ. Warner Swasey Obs., 1,1

Turnshek D. A., Bohlin R. C., Williamson II R. L., Lupie O. L., Koornneef J., Morgan D. H., 1990, ApJ, 99, 1243

Vink J. S., 2018, A\&A, 619, A54

Vink J. S., de Koter A., Lamers H. J. G. L. M., 1999, A\&A, 350, 181

Wampler E. J., Wang L., Baade D., Banse K., D'Odorico S., Gouiffes C., Tarenghi M., 1990, ApJ, 362, L13

Wegner W., 2014, Acta Astron., 64, 261

Werner M. W. et al., 2004, ApJS, 154, 1

Wright E. L. et al., 2010, AJ, 140, 1868

Zinn J. C., Pinsonneault M. H., Huber D., Stello D., 2019, ApJ, 878,136 